\title{
DEFECTOS REFRACTIVOS EN UNA POBLACIÓN INFANTIL ESCOLARIZADA EN BOGOTÁ DC
}

Adriana Solano MD*, Fernando Schoonewolff MD**, María Paula Hernández MD***, Miriam Ibeth Isaza MD***

\section{Resumen}

Objetivo: describir la frecuencia y distribución de los diferentes tipos de errores refractivos en una población infantil escolarizada de Bogotá DC. Metodología: estudio de corte transversal que incluyó niños escolares de un colegio de Bogotá DC hasta segundo grado de primaria, cuyos padres o acudientes firmaron el consentimiento informado. La valoración fue realizada por residentes de oftalmología y optómetras del Hospital de San José, con ayuda de un autorrefractómetro para buscar la prevalencia de defectos refractivos. Resultados: se analizaron 109 niños entre 4 y 9 años, encontrándose la mayoría entre 6 y 7 años. El defecto refractivo esférico más frecuente fue astigmatismo $(15,1 \%)$, seguido por hipermetropía $(13,8 \%)$ y miopía (2,3\%). Hubo sospecha de ambliopía (agudeza visual corregida peor de 20/40) en el 21,5\% de la población. El 11\% presentó estrabismo, encontrando 9 casos de exotropia y 3 de endotropia. Conclusiones: se encontró un alto porcentaje de niños con disminución de la agudeza visual, siendo muy pocos los que han recibido una valoración oftalmológica u optométrica previas a pesar de ser obligatorias en el país. Se insiste en la necesidad de vigilar el cumplimiento estricto de los programas gubernamentales de salud visual en Bogotá DC y en el resto del país.

Palabras clave: defecto refractivo, población escolarizada, hipermetropía, miopía, astigmatismo, ambliopía, estrabismo.

\section{REFRACTIVE ERRORS IN CHILDREN ATTENDING SCHOOL IN BOGOTÁ}

\section{Abstract}

Objective: to describe the frequency and distribution of different types of refractive errors in a child population attending school in Bogotá DC. Methodology: cross-sectional study including children attending a school in Bogotá DC up to the second grade level, whose parents and guardians signed the informed consent. This assessment was delivered by ophthalmology residents and optometrists of Hospital de San José, aided by an auto refractometer in order to find the prevalence of refractive errors. Results: 109 boys/girls aged between 4 and 9 years were tested, most of them were aged between 6 and 7 years. The most common spherical refractive error found was astigmatism (15.1\%), followed by hypermetropia (13.8\%) and myopia (2.3\%). Amblyopia was suspected (corrected visual acuity lower than 20/40) in $\mathbf{2 1 . 5 \%}$ of the study population. $\mathbf{1 1} \%$ presented strabismus with 9 cases of exotropia and 3 of endotropia. Conclusions: a high percentage of children with impaired visual acuity were identified. Very few of them had received a previous ophthalmologic or optometric assessment despite its obligatory status in the country. It is emphasized that governmental visual heath programs must be fulfilled in Bogotá DC and the rest of the country.

Key words: refractive error, school population, hypermetropia, myopia, astigmatism, amblyopia, strabismus.

Fecha recibido: mayo 2 de 2011 - Fecha aceptado: junio 10 de 2011

Médica Cirujana, Oftalmóloga,Supraespecialista en Oftalmologia Pediátrica y Estrabismo. Instructor Asociado servicio de Oftalmología Hospital de San José, Fundación Universitaria de Ciencias de la Salud. Bogotá, DC Colombia.
** Médico Cirujano Residente. IV de Oftalmología. Fundación Universitaria de Ciencias de la Salud, Hospital de San José. Bogotá, DC Colombia

* Oftalmóloga. Egresada de la Fundación Universitaria de Ciencias de la Salud, Hospital de San José. Bogotá, DC Colombia. 


\section{Introducción}

Los defectos de refracción son de gran importancia durante la infancia porque causan disminución de la agudeza visual, impidiendo que el niño tenga una imagen nítida que le permita lograr un desarrollo normal de la función visual; las consecuencias son variadas y una de las más importantes es la ambliopía. ${ }^{1-4} \mathrm{El}$ error refractivo puede ser diagnosticado, cuantificado y corregido en forma fácil. ${ }^{4,5}$ Esta última no requiere grandes sumas de dinero y debe ser parte integral del alcance del cuidado del ojo. ${ }^{5}$ Es así como los estudios de tamizaje son necesarios para motivar la detección temprana y el tratamiento de los defectos refractivos en la población escolar. Dicha detección tiene por lo regular un resultado satisfactorio, no existen mayores dificultades para la aplicación de las pruebas, el seguimiento es fácil y las intervenciones son eficaces en la mayoría de los casos. ${ }^{3-5}$

La omisión de este cuidado tiene consecuencias negativas para la vida, en términos de pérdida de oportunidades de educación y desarrollo en los niños, de empleo, productividad y calidad de vida en los adultos, involucrando no solo al individuo sino también a la familia y a la sociedad. ${ }^{3-5}$ Existe desde julio de 2005 en Bogotá un acuerdo que tiene como fin que los niños de estratos 1 y 2 (que se encuentran bajo el régimen subsidiado de salud en su mayoría), tengan la oportunidad de tamizajes tanto visuales como auditivos para de esta forma poder mejorar la calidad de vida y darles oportunidades para su futuro. ${ }^{6}$ Según datos reportados por este acuerdo, sólo el $36,4 \%$ de las entidades territoriales que tienen programas de salud en las escuelas distritales realizan tamizajes de agudeza visual y auditiva a sus estudiantes. ${ }^{6}$

Existen varios reportes epidemiológicos realizados alrededor del mundo evaluando la prevalencia de defectos refractivos en poblaciones escolares, tales como Corea del Sur, ${ }^{7}$ Irán, ${ }^{8}$ China,,${ }^{9-11}$ Nepal, ${ }^{12}$ y Chile. ${ }^{13,14} \mathrm{La}$ mayor parte de Latinoamérica carece de datos acerca de la prevalencia de defectos refractivos con solo tres estudios a gran escala publicados. Estos fueron realizados en La Florida, Chile, ${ }^{14}$ Ciudad de México ${ }^{15}$ y Bogotá, DC, Colombia. ${ }^{16} \mathrm{El}$ primero reportó que causaban el $56,3 \%$ de los casos de visión reducida y que el $7 \%$ de los niños se beneficiarían de corrección óptica que no disponían en el momento. En el segundo se evaluó solo la presencia de miopía, encontrándola en el 33\% de la población estudiada. En el trabajo realizado en Colombia se identificó una prevalencia de hipermetropía de $59,2 \%$, astigmatismo $28,2 \%$, miopía $4 \%$ y ningún defecto refractivo (emetropía) en el $9 \%$ de los niños. Este estudio fue hecho en el año 2000 y se evaluaron 1.250 estudiantes de colegios públicos y privados, con el objetivo de describir alteraciones oculares visuales. Se halló una prevalencia de defecto refractivo del $42 \%$ en niños en edad escolar en Bogotá DC, entre los cuales más del $60 \%$ de los afectados no usaban gafas. ${ }^{16}$ Otro estudio realizado en San Diego con un $74 \%$ de población latina, reportó 7,5\% de hipermetropía, $3 \%$ miopía y $5,5 \%$ astigmatismo. ${ }^{17}$ Cabe aclarar que las definiciones de estos errores de refracción no son iguales en todos los estudios. Por todo esto existe la necesidad de realizar investigaciones para conocer la frecuencia de los errores de refracción, para mejorar las estrategias de detección temprana y tratamiento oportuno. El objetivo de este trabajo es describir la frecuencia y distribución de los errores refractivos en una población infantil escolarizada de Bogotá DC.

\section{Métodos}

El protocolo para este estudio fue aprobado por el comité de ética médica del Hospital de San José. Se invitó a todos los niños matriculados en un colegio distrital de Bogotá DC, mediante una reunión de padres de familia en donde se informó a los profesores del colegio y a los padres o acudientes sobre las características de la jornada y su fin. Después se envió una circular invitando a participar en el estudio. En esta circular se explicaba la importancia de realizar un tamizaje de agudeza visual y se solicitaba la autorización de la evaluación (incluyendo la aplicación de medicamentos cicloplégicos).

Se incluyeron todos los niños entre cuatro y diez años que se encontraran cursando los grados 0,1 y 2 de la jornada matutina y vespertina del colegio, cuyos padres o acudientes dieran consentimiento informado para el 
examen. Los criterios de exclusión eran aquellos con antecedente de síndrome convulsivo, Down, parálisis espástica, lesiones cerebrales y albinos. Se enviaron en total 204 circulares, 108 niños cumplieron los criterios y fueron estudiados, por lo cual no se calculó el tamaño de la muestra.

El examen oftalmológico se realizó en tres jornadas en días diferentes. La primera consistió en la recolección de datos en las instalaciones del colegio, en donde dos personas (residente de oftalmología y profesora o asistente encargado del curso a evaluar) tomaron los datos personales del estudiante en el formato, previa verificación del adecuado diligenciamiento del consentimiento informado y confirmación de la ausencia de criterios de exclusión.

En la segunda jornada dos residentes de primer y tercer año de oftalmología midieron tanto la agudeza visual lejana como la próxima. Esto se hizo en un salón del colegio en donde se realizó el estudio, que debía tener una longitud de seis metros para una adecuada toma de la agudeza visual lejana sin corrección (AVLSC) y luego con corrección (AVLCC). Estas se tomaron utilizando la cartilla de Snellen de figuras, primero en el ojo derecho (OD) y luego en el ojo izquierdo (OI), con la utilización de un oclusor. También se valoró la agudeza visual próxima sin corrección (AVPSC) con una cartilla de Allen a $33 \mathrm{~cm}$ de distancia, primero en OD y luego en OI con la utilización de un oclusor. Luego se evaluó la presencia de estrabismo mediante examen de motilidad ocular y cover test, este último se midió como se ha estandarizado para la práctica clínica usual y fue realizado en todos los casos por un residente de último año de oftalmología. Por último, se hizo la medición del punto próximo de convergencia.

La tercera jornada (cicloplegia y autoqueratorrefracción) se efectuó unos días después de la segunda en los salones del curso a evaluar con la colaboración de la profesora encargada. Se verificó la firma del consentimiento informado y luego se aplicó a cada estudiante que no tuviera criterios de exclusión una gota de anestésico tópico proparacaína 1\% (AlcaineÒ) y después una gota de tropicamida $1 \%$ (MydriacylÒ) con ciclopentolato 1\% (CiclogylÒ) en cada ojo. A los
40 minutos se realizó la autoqueratorrefracción en OD y OI, con el autoqueratorrefractómetro marca Huvitz MRK 3100, el cual fue operado por una persona entrenada y muy familiarizada con su funcionamiento, quien realizó tres tomas en cada ojo. Después se practicó una prueba subjetiva de la agudeza visual lejana corregida con la misma cartilla de Snellen, para determinar si había o no sospecha de ambliopía (peor de 20/40). $\mathrm{Al}$ identificar los pacientes con defectos refractivos, ambliopía o estrabismo se diligenció una solicitud de autorización de servicios de carácter prioritario para una valoración por oftalmología pediátrica. Esta solicitud fue entregada a cada estudiante para que fuera llevada a la oficina del servicio de salud (EPS o ARS), con el fin de que el niño tuviera una valoración y tratamiento oportuno.

La definición de defecto refractivo utilizada en este trabajo fue tomada según los resultados del Vision in Preschoolers Study, ${ }^{18}$ en el cual se considera importante en la clínica detectar y corregir con lentes ${ }^{4}$ la miopía mayor de una dioptría (D) en mayores de cuatro años, la hipermetropía superior de tres D en mayores de cuatro años y el astigmatismo mayor de $1.50 \mathrm{D}$ en el mismo grupo de edad. Se consideró la anisometropía como la diferencia mayor de $2.00 \mathrm{D}$ entre los dos ojos. La emetropía se definió como los errores refractivos que se encuentran por debajo de estos niveles. El análisis estadístico de los datos fue realizado con estadísticas descriptivas, frecuencias absolutas y relativas, medidas de tendencia central y de dispersión, utilizando los programas Microsoft Excel 2003 y Stata 10.

\section{Resultados}

Se analizaron un total de 204 niños, logrando obtener datos de cicloplegia y consentimiento informado en 109. El 16,5\% tenía entre 4 y 5 años, el $67,9 \%$ entre 6 y 7 y el 15,6\% entre 8 y 9 años. El 47,7\% fueron de género femenino. Algunos datos de estrato socioeconómico, antecedente familiar o examen previo no se tomaron, ya que ni los niños ni sus acudientes conocían esta información (Tabla 1).

El 37,4\% de los OD y el 38,3\% de los OI presentaron agudeza visual lejana sin corrección de 20/20 (Figura 
1). Este porcentaje aumentó a $44,8 \%$ en el OD y $47,6 \%$ en el OI con la corrección según la autorrefracción bajo cicloplegia. Sin el uso de corrección el 76,7\% y $80,4 \%$ de los OD y OI respectivamente presentaron agudeza visual mejor o igual a 20/40.

De los OD con miopía el $66,7 \%$ fue en mujeres y el $33,3 \%$ en hombres, mientras que en los hipermetrópicos hubo más hombres $(62,5 \%)$ que mujeres $(37,5 \%)$. En el OI la miopía se distribuyó de manera igual en los dos géneros $(50 \%)$ y hubo más ojos hipermétropes en hombres $(64,7 \%)$. En los OD que presentaron astigmatismo $53,3 \%$ eran de sexo masculino y $46,7 \%$ femenino, hallazgos similares a los encontrados en el OI con 55,6\% hombres y el $44,4 \%$ mujeres (Tabla 1).

Con respecto a los defectos de refracción, la mayoría de los niños estudiados fueron emétropes $(84,4 \%$ en el OD y $83,5 \%$ en el OI), según la definición empleada en este estudio. El resto presentaron algún tipo de defecto refractivo, encontrando una mayor prevalencia de astigmatismo ambliogénico $(13,8 \%$ OD, $16,5 \%$ OI), seguido de hipermetropía $(12,8 \%$ OD, $14,7 \%$ OI) y por último miopía $(2,8 \%$ OD, $1,8 \%$ OI). Los resultados de agudeza visual y refracción están dados en la Tabla 2. En el total de la población, la mediana de la agudeza visual lejana (AVL) fue de 0.66 (RIQ: 0.50-1.00), con iguales resultados en ambos ojos (Tabla 2).

La mitad de los pacientes que presentaron emetropía en el ojo derecho tuvieron AVL de 0.66 (RIQ: 0.501.00 ), seguidos por los hipermétropes con 0.40 (RIQ: 0.28-0.50), los que se clasificaron como astigmatismo 0.34 (RIQ 0.20-0.50), y por último los miopes 0.19 (RIQ: 0.08-0.39) (Tabla 2).

En la Figura 2 se presenta la distribución de los equivalentes esféricos de ambos ojos con media de 1.2 D (DE: 1.4) en el OD y 1.3 (DE: 1.3) en el OI. Los equivalentes esféricos se encontraron entre el rango de

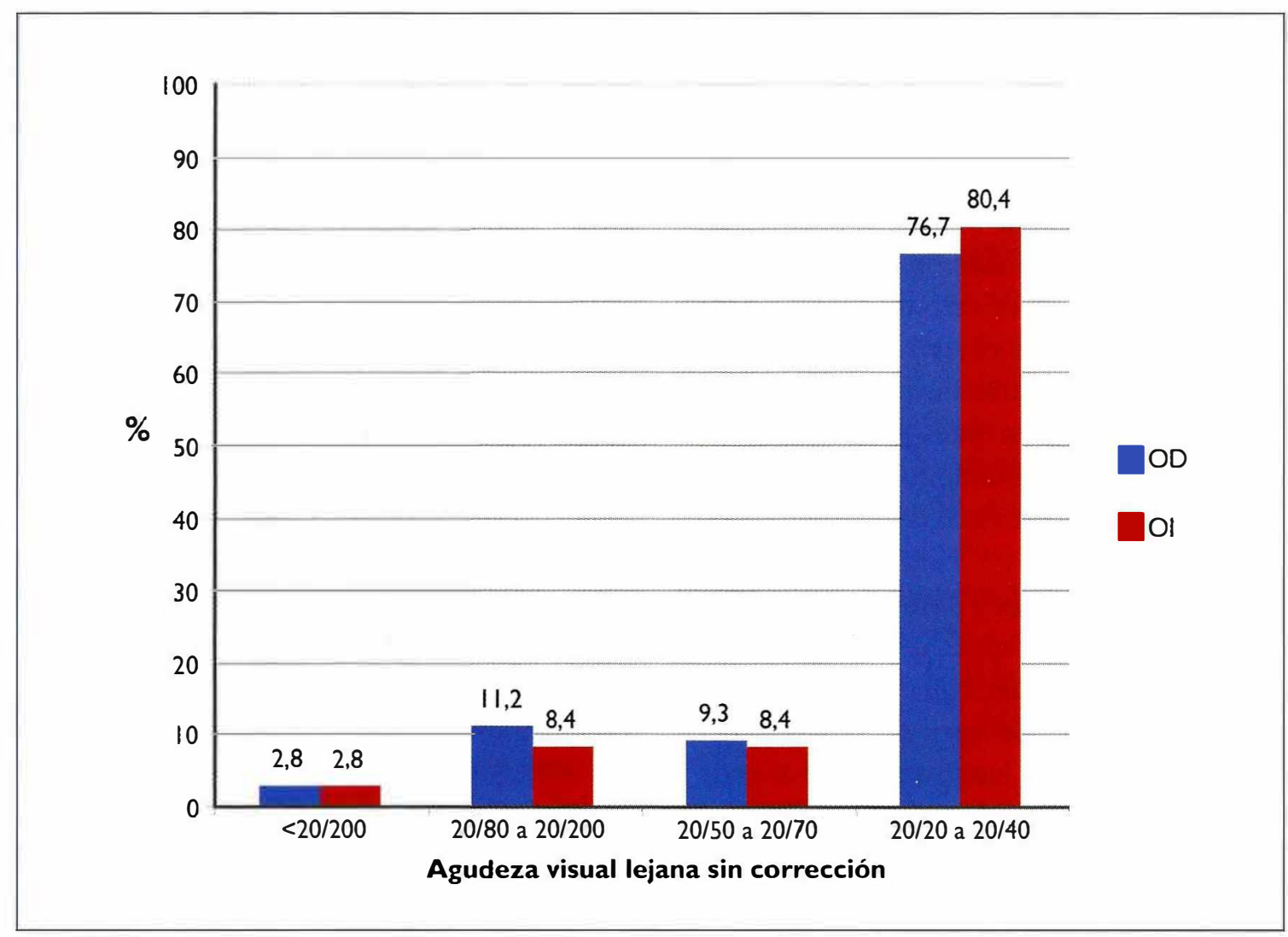

Figura I. Distribución de la agudeza visual en ojos derecho e izquierdo. 
Tabla I. Caracteristicas demográficas por grupo de edad

\begin{tabular}{|c|c|c|c|c|c|}
\hline Edad & $4-5 *(n=18)$ & $6-7 *(n=74)$ & $8-10 *(n=17)$ & Total $(n=109)$ & $\%$ \\
\hline \multicolumn{6}{|l|}{ Género $n=109$} \\
\hline femenino/masculino & $9 / 9$ & $35 / 39$ & $8 / 9$ & $52 / 57$ & $48 / 52$ \\
\hline \multicolumn{6}{|l|}{ Estrato $n=89$} \\
\hline 1 & 11 & 22 & 4 & 37 & 33.9 \\
\hline 2 & 2 & 27 & 9 & 38 & 34.9 \\
\hline 3 & 5 & 9 & 0 & 14 & 12.8 \\
\hline sin datos & 0 & 16 & 4 & 20 & 18.3 \\
\hline \multicolumn{6}{|c|}{ Antecedente familiar $n=90$} \\
\hline sí & 6 & 27 & 3 & 36 & 33.0 \\
\hline no & 12 & 32 & 10 & 54 & 49.5 \\
\hline sin datos & 0 & 15 & 4 & 19 & 17.4 \\
\hline \multicolumn{6}{|l|}{ Estrabismo $n=109$} \\
\hline sí - & 2 & 6 & 4 & 12 & 11.0 \\
\hline no & 16 & 68 & 13 & 97 & 89.0 \\
\hline \multicolumn{6}{|l|}{ Uso de gafas $n=91$} \\
\hline sí & 0 & 2 & 0 & 2 & 1.8 \\
\hline no & 18 & 58 & 13 & 89 & 81.7 \\
\hline sin datos & 0 & 14 & 4 & 18 & 16.5 \\
\hline \multicolumn{6}{|c|}{ Examen previo $n=90$} \\
\hline sí & 2 & 3 & 1 & 6 & 5.5 \\
\hline no & 16 & 56 & 12 & 84 & 77.1 \\
\hline $\sin$ datos & 0 & 15 & 4 & 19 & 17.4 \\
\hline \multicolumn{6}{|c|}{ Defecto refractivo $n=218 \mathrm{OD} / \mathrm{O}+\dagger$} \\
\hline emetropía & $17 / 17$ & $61 / 61$ & $14 / 13$ & $92 / 91$ & $84.4 / 83.5$ \\
\hline miopía & $1 / 0$ & $2 / 1$ & $0 / 1$ & $3 / 2$ & $2.8 / 1.8$ \\
\hline hipermetropía & $0 / 1$ & $11 / 12$ & $3 / 3$ & $14 / 16$ & $12.8 / 14.7$ \\
\hline astigmatismo & $0 / 2$ & $12 / 14$ & $3 / 2$ & $15 / 18$ & $13.8 / 16.5$ \\
\hline
\end{tabular}

* Edad en años. † Ojo Derecho/Ojo Izquierdo.

Tabla 2. Características clínicas de los niños con defectos refractivos

\begin{tabular}{|c|c|c|c|c|c|}
\hline $\begin{array}{l}\text { Mediana } \\
\text { (RIQ) } \dagger\end{array}$ & $\begin{array}{c}\text { Miopia } \\
\text { (OD/OI* } n=4 / 2)\end{array}$ & $\begin{array}{l}\text { Hipermetropia } \\
\left(O D / O{ }^{*} n=16 / 17\right)\end{array}$ & $\begin{array}{c}\text { Astigmatismo } \\
\left(\mathrm{OD} / \mathrm{O}{ }^{*} \mathrm{n}=\mid \mathrm{5} / 18\right)\end{array}$ & $\begin{array}{c}\text { Emetropia } \\
\text { (OD/OI* } n=89 / 90)\end{array}$ & $\begin{array}{c}\text { Total } \\
\text { (OD/OI* } n=109 / 109)\end{array}$ \\
\hline Esfera OD $\nmid$ & $-1.65(-2.63 a-1.25)$ & $+3.88(+3.38 \mathrm{a}+4.75)$ & $+2.25(+0.50 a+4.50)$ & $+1.25(+0.75 a+1.75)$ & $+1.25(+0.75 a+2.25)$ \\
\hline Cilindro OD & $-1.88(-2.50 a-0.88)$ & $-1.38(-2.25 a-0.50)$ & $-2.50(-3.50 a-1.75)$ & $-0.50(-0.75$ a 0.00$)$ & $-0.50(-1.00 a-0.25)$ \\
\hline $\mathrm{EE} \neq \mathrm{OD} \dagger$ & $-2.50(-3.44 a-2.13)$ & $+3.38(+2.88 \mathrm{a}+3.88)$ & $+1.38(-0.38 \mathrm{a}+3.38)$ & $+0.94(+0.50 a+1.50)$ & $+1.00(+0.63 a+1.75)$ \\
\hline $\operatorname{AV} \oint O D \dagger$ & $0.19(0.08$ a 0.39$)$ & $0.40(0.28$ a 0.50$)$ & $0.34(0.20 \mathrm{a} 0.50)$ & $0.66(0.50$ a 1.00$)$ & $0.66(0.50$ a 1.00$)$ \\
\hline Esfera OI $\dagger$ & $-1.88(-2.50 a-1.25)$ & $+3.75(+3.5 a+5.25)$ & $+2.63(+1.75 a+3.75)$ & $+1.25(+1.00 a+1.75)$ & $+1.50(+1.00 a+2.25)$ \\
\hline Cilindro $\mathrm{Ol} \uparrow$ & $-2.63(-3.00 a-2.25)$ & $-1.50(-2.75$ a -0.75$)$ & $-2.75(-3.00 a-2.00)$ & $-0.50(-0.75 a-0.25)$ & $-0.50(-1.00$ a -0.25$)$ \\
\hline $\mathrm{EE} \neq \mathrm{OI} \dagger$ & $-3.19(-3.63$ a -2.75$)$ & $+3.50(+2.75 a+3.75)$ & $+1.32(+0.13 a+2.63)$ & $+1.00(+0.50 a+1.38)$ & $+1.13(+0.75 a+1.75)$ \\
\hline AVફ̧OI $\dagger$ & $0.15(0.02$ a 0.28$)$ & $0.45(0.28$ a 0.58$)$ & $0.40(0.28$ a 0.50$)$ & $0.66(0.50$ a 1.00$)$ & $0.66(0.50$ a 1.00$)$ \\
\hline
\end{tabular}

* Ojo derecho/ojo izquierdo. † Mediana (RIQ: rango intercuartílico). ‡Equivalente esférico. § Agudeza visual. 


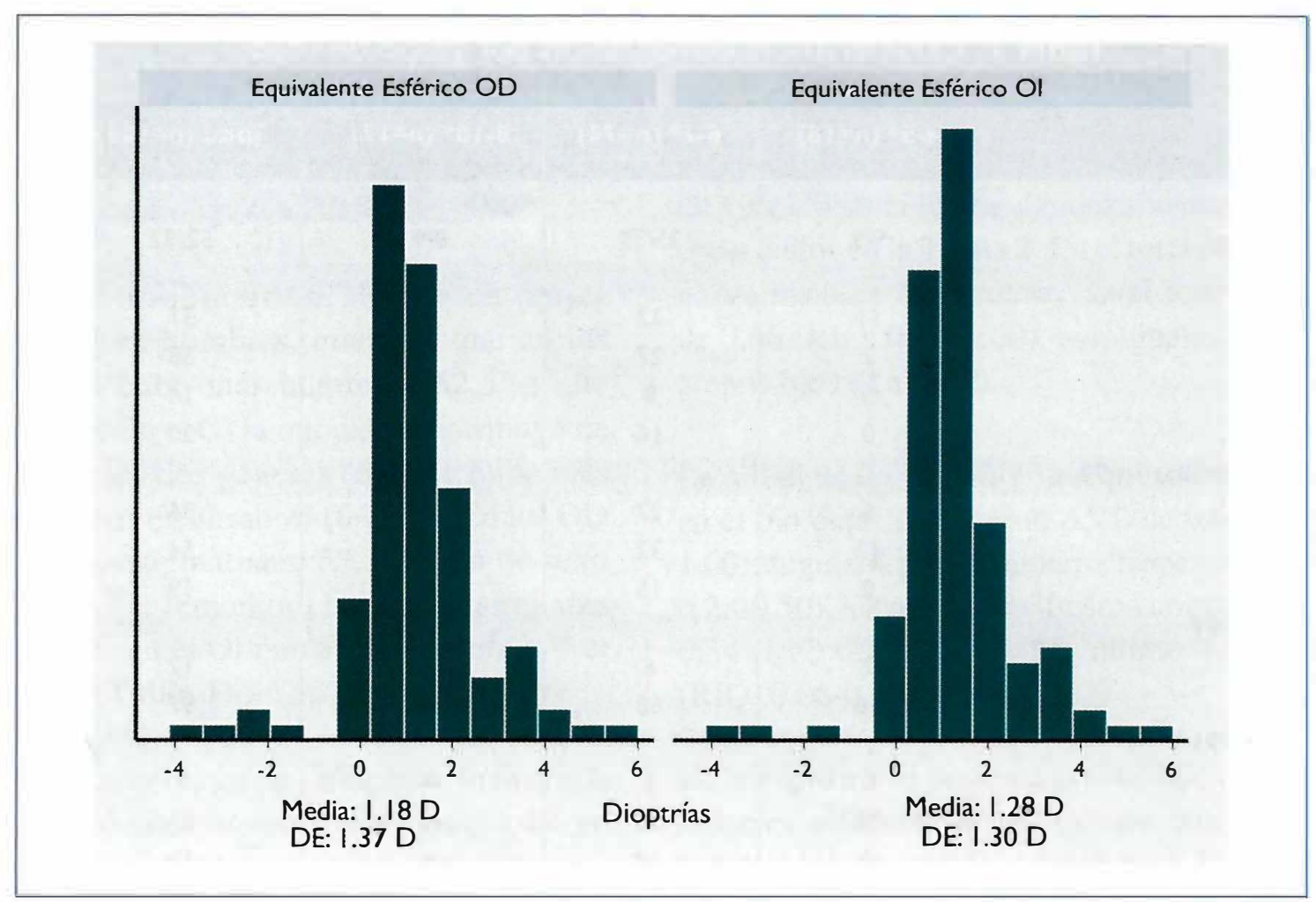

Figura 2. Distribución de los equivalentes esféricos en ojos derecho e izquierdo.

emetropía (-1.00 a +3.00) en la mayoría de los pacientes en ambos ojos, con el $83,5 \%$ en el OD y el $86,6 \%$ en el OI. Con respecto al estrabismo, se encontraron doce casos en el estudio, $33,3 \%$ en mujeres y $66,7 \%$ en hombres. De estos, nueve niños no usaban corrección y tres no tenían datos. En estos casos predominó la exotropía con nueve casos y se observaron tres casos de endotropía.

Hubo sospecha de ambliopía (agudeza visual lejana corregida o AVLC peor de 20/40) en el OD en el 23,4\% y en OI en el 19,6\% de la población. La mitad de los pacientes con ambliopía tuvieron una AVLC de 0.28 (RIQ: 0.20-0.40), muy similar a la mediana de los pacientes con antimetropía [0.28 (RIQ: 0.05-0.50)]. Los que presentaron anisometropía también tuvieron una peor visión que los de la población total, con mediana de la agudeza visual de 0.50 (RIQ: 0.10-0.50) (Tabla 3). La frecuencia de antecedente familiar de defecto refractivo fue muy similar entre los grupos de edad y se distribuyó de manera uniforme entre los diferentes defectos refractivos.

\section{Discusión}

La mayoría de los niños estudiados estaban entre seis y siete años, lo cual es importante ya que en ese momento está por finalizar el proceso de maduración visual y todavía existe la posibilidad de tratamiento para corrección de cualquier defecto refractivo, estrabismo y ambliopía. ${ }^{1,2}$ Cabe aclarar que los resultados de estos tratamientos no serán tan buenos, en especial en ambliopía y que éstos son efectivos entre menor sea el niño cuando sean instaurados.

Es importante destacar que la distribución de defectos refractivos en nuestra población fue similar a la encontrada en otros estudios como los de Bogotá DC, ${ }^{16} \mathrm{México}^{15}$ (en donde solo midieron la presencia de miopía), San Diego ${ }^{17}$ (con el 74\% de la población siendo latina) y La Florida (Chile) ${ }^{14}$ El defecto refractivo más prevalente en todos los grupos de edad y en los dos sexos fue el astigmatismo con $15,1 \%$ (27\% en el estudio realizado en Chile, $5,5 \%$ en San Diego y 28,2\% en Bogotá), seguido por hipermetropía con $13,8 \%$, y miopía $2,3 \%$ de la 


\section{Tabla 3. Características clínicas de los niños con anisometropía, antimetropía y sospecha de ambliopía}

\begin{tabular}{|c|c|c|c|}
\hline n/Total & $\begin{array}{l}\text { Anisometropia* } \\
(5 / 109)\end{array}$ & $\begin{array}{l}\text { Antimetropia* } \\
(2 / 109)\end{array}$ & $\begin{array}{c}\text { Sospecha ambliopia } \\
\text { (I } 7 / 107)\end{array}$ \\
\hline Esfera OD $†$ & $-1.00(-1.75 a+2.50)$ & $-2.63(-3.50$ a -1.75$)$ & $+2.25(+1.50 \mathrm{a}+4.50)$ \\
\hline Cilindro OD † & $-1.25(-1.50$ a -1.25$)$ & $-0.88(-1.25$ a -0.50$)$ & $-1.25(-1.75$ a -0.50$)$ \\
\hline EE $\ddagger$ OD $†$ & $-2.00(-2.25 \mathrm{a}+1.88)$ & $-3.07(-4.13$ a -2.00$)$ & $+2.00(+0.75 \mathrm{a}+3.38)$ \\
\hline AV§ OD† & $0.50(0.10$ a 0.50$)$ & $0.28(0.05$ a 0.50$)$ & $0.28(0.20$ a 0.40$)$ \\
\hline Esfera OI† & $+0.50(+0.50 \mathrm{a}+1.75)$ & $+0.50(+0.50 \mathrm{a}+0.50)$ & $+2.50(+1.75 a+5.25)$ \\
\hline Cilindro OI $t$ & $-2.25(-3.00 a-1.75)$ & $-0.88(-1.75$ a 0.00$)$ & $-1.50(-2.25$ a -0.50$)$ \\
\hline EE $\ddagger$ OI† & $+0.13(-0.38 \mathrm{a}+0.50)$ & $+0-06(-0.38 \mathrm{a}+0.50)$ & $+2.00(+1.25 \mathrm{a}+3.75)$ \\
\hline AV§ OI† & $0.40(0.05$ a 0.66$)$ & $0.53(0.40$ a 0.66$)$ & $0.40(0.28$ a 0.50$)$ \\
\hline
\end{tabular}

* Pacientes afectados/pacientes evaluados. † Mediana (RIQ: rango intercuartílico). ‡ Equivalente esférico. § Agudeza visual.

población (7,3\% Chile, 33\% México, 3\% San Diego y $4,0 \%$ Bogotá). ${ }^{14-17}$

Cabe resaltar que el estudio chileno evaluó 5.303 pacientes entre los cinco y quince años de edad y que también tuvieron una definición diferente de los defectos refractivos. En ese estudio la miopía se catalogó como la esfera mayor de -0.5 , y la hipermetropía como mayor de +2.00 . También definieron el astigmatismo como un cilindro mayor de 0.75 dioptrías. Esto da un rango menor a la emetropía y podría en parte explicar la diferencia en los resultados. En el estudio colombiano se evaluaron 1.250 niños, entre los cinco y catorce años, no se especifica cómo se clasificaron los defectos refractivos, algo que si se explica en nuestro trabajo. En el realizado en San Diego, la definición fue igual a la nuestra y se evaluaron 507 niños entre los tres y los cinco años de edad.

De igual manera observamos que la mayoría de los niños presentan una AVL mejor de 20/40 en AO, la cual es considerada como aceptable según los lineamientos de la Organización Mundial de la Salud (OMS). ${ }^{4}$ Esto se traduce en que el $76,7 \%$ de los niños de este estudio podría tener un desempeño escolar adecuado y una productividad y calidad de vida aceptables durante la adultez, aunque una visión menor de 20/20 no les permitirá ser aptos para múltiples ocupaciones y profesiones que exigen una visión perfecta.

A pesar de los resultados encontrados y de las políticas de salud visual de nuestro país, solo el 2,2\% de los niños de nuestro estudio usaba corrección en el momento del examen y al $6,7 \%$ se les había realizado un examen oftalmológico u optométrico previo. De la misma forma hallamos que la mayoría de los niños pertenecía a alguno de los regímenes de salud, lo que debería permitir el acceso a una valoración por oftalmología desde edades tempranas, pero por los resultados aquí encontrados consideramos que esto no se cumple. Se remitieron el 33,3\% a su respectivo seguro de salud. Los que no tenían ninguno se pusieron en contacto con las profesoras para indicarles los trámites para la afiliación.

A diferencia de lo reportado en la literatura, que muestra como durante el proceso de emetropización disminuye la prevalencia de hipermetropía en la población infantil, ${ }^{1,2}$ en nuestro trabajo la cantidad de niños hipermétropes fue menor en los más pequeños. La miopía fue más común en los niños mayores, menores, que va de acuerdo con lo reportado en estudios similares. ${ }^{13,18}$

Con respecto al instrumento de medición utilizado sabemos que estudios anteriores han demostrado la efectividad del uso de autoqueratorrefractómetros similares en niños mayores de tres años, mostrandouna sensibilidad del $88 \%$ y una especificidad del $58 \% .^{19,20}$

Por último, estamos conscientes que una limitación importante de este estudio es la población tan pequeña y que todos los niños examinados son del mismo colegio. Esto no permite extrapolar los resultados 
al resto de la población. Podemos concluir que son necesarios estudios más grandes para establecer la verdadera distribución de los defectos refractivos en la población escolar de nuestro país y que es de vital importancia para el futuro de la nación evaluar y tratar esta patología, en especial en poblaciones vulnerables como la estudiada, pues se ha demostrado que el tratamiento (la prescripción y el uso de anteojos) es no solo efectivo sino también económico y fácil de suministrar. ${ }^{3}$

\section{Referencias}

1. Marroquín G, Solano A. Ambliopía. In: Marroquín G, editor. Oftalmología Pediátrica - Guías de Manejo. 1 ed. Bogotá: Icono Editorial. 2006;153-66.

2. ZorabR, Straus H, et al. Amblyopia. In:Zorab R, Straus H, et al., editors. Pediatric Ophthalmology and Strabismus. American Academy Of Ophthalmology. 2006; 63-79.

3. Roch-Levecq AC, Brody BL, Thomas RG, Brown SI. Ametropia, preschoolers' cognitive abilities, and effects of spectacle correction. Arch Ophthalmol. 2008 Feb;126(2):252-8.

4. Hartmann EE, Dobson V, Hainline L, Marsh-Tootle W, Quinn GE, Ruttum MS et al. Preschool vision screening: summary of a Task Force report. Behalf of the Maternal and Child Health Bureau and the National Eye Institute Task Force on Vision Screening in the Preschool Child. Pediatrics. 2000 Nov;106(5):1105-16.

5. Pizzarello L, Abiose A, Ffytche T, Duerksen R, Thulasiraj R, Taylor H, et al VISION 2020: The Right to Sight: a global initiative to eliminate avoidable blindness. Arch Ophthalmol. 2004 Apr;122(4):615-20.

6. Acuerdo 247 de 2005 , Un compromiso social contra la pobreza y la exclusión, 247 de 2005, Concejo de Bogotá D.C., 2005.

7. Lim HT, Yu YS, Park SH, Ahn H, Kim S, Lee M, et al. The Seoul Metropolitan Preschool Vision Screening Progranume: results from South Korea. Br J Ophthalmol. 2004 Jul;88(7):929-33.
8. Yekta A, Fotouhi A, Hashemi H, Dehghani C, Ostadimoghaddam H, Heravian J, et al. Prevalence of refractive errors among schoolchildren in Shiraz, Iran. Clin Experiment Ophthalmol. 2010 Apr;38(3):242-8

9. Negrel AD, Maul E, Pokbarel GP, Zhao J, Ellwein LB. Refractive Error Study in Children: sampling and measurement methods for a multi-country survey. Am J Ophthalmol. $2000 \mathrm{Apr} ; 129(4): 421-6$.

10. Zhao J, Pan X, Sui R, Munoz SR, Sperduto RD, Ellwein LB. Refractive Error Study in Children: results from Shunyi District, China. Am J Ophthalmol. 2000 Apr;129(4):427-35.

11. Pi LH, Chen L, Liu Q, Ke N, Fang J, Zhang S, et al. Refractive status and prevalence of refractive епог in suburban school-age children. Int J Med Sci. 2010 Oct $18 ; 7(6): 342-53$

12. PokharelGP, Negrel AD, Munoz SR, Ellwein LB. Refractive Error Study in Children: results from Mechi Zone, Nepal. Am J Ophthalmol. 2000 Apr;129(4):436-44.

13. Gilbert CE, Ellwein LB. Prevalence and causes of functional low vision in school-age children: results from standardized population surveys in Asia, Africa, and Latin America. Invest Ophthalmol V is Sci. 2008 Mar;49(3):877-81.

14. Maul E, BarrosoS, Munoz SR, Sperduto RD, Ellwein LB. Refractive Error Study in Children: results from La Florida, Chile. Am J Ophthalmol. 2000 Apr;129(4):445-

15. Rodríguez-Abrego G, Sotelo-Duenas HM. [Myopia prevalence among schoolage children in a suburban population]. Rev Med Inst Mex Seguro Soc. 2009 Jan; $47(1): 39-44$

16. Hernández Luna CP, Barrera Santos DC, Guiza Segura C, Rodríguez Malagón JP, Ludeman WE, Gómez Montaña SP. Estudio de prevalencia en salud visual en una población escolar de Bogotá, Colombia, 2000. Cienc Tecnol Salud Vis Ocul. 2003 Sep;(1):11-23

17. Brody BL, Roch-Levecq AC, Klonoff-Cohen HS, Brown SI. Refractive errors in low-income preschoolers. Ophthalmic Epidemiol. 2007 Jul;14(4):223-9.

18. Kulp MT. Findings from the Vision in Preschoolers (VIP) Study. Optom Vis Sci. 2009 Jun;86(6):619-23.

19. Kemper AR, Keating LM, Jackson JL, Levin EM. Comparison of monocular autorefraction to comprehensive eye examinations in preschool-aged and younger children. Arch Pediatr Adolesc Med. 2005 May;159(5):435-9.

20. Prabakaran S, Dirani M, Chia A, Gazzard G, Fan Q, Leo SW, et al. Cycloplegic refraction in preschool children: comparisons between the hand-held autorefractor, table-mounted autorefractor and retinoscopy. Ophthalmic Physiol Opt. 2009 Jul;29(4):422-6 